\title{
Hole-doping effects on a frustrated spin ladder
}

\author{
Akira Kawaguchi, Akihisa Koga, Kouichi Okunishi ${ }^{1}$ and Norio Kawakami \\ Department of Applied Physics, Osaka University, Suita, Osaka 565-0871, Japan \\ ${ }^{1}$ Department of Physics, Niigata University, Igarashi 2, Niigata 950-2181 Japan
}

(Dated: November 14, 2018)

\begin{abstract}
Hole-doping effects are investigated on the $t$ - $J$ ladder model with the linked-tetrahedra structure. We discuss how a metal-insulator transition occurs upon hole doping with particular emphasis on the effects of geometrical frustration. By computing the electron density and the spin correlation function by the density matrix renormalization group, we show that strong frustration triggers a first-order transition to a metallic phase, when holes are doped into the plaquette-singlet phase. By examining spin excitations in a metallic case in detail, we discuss whether the spin-gap phase persists upon hole doping according to the strength of frustration. It is further shown that the lowest excited state in a spin-gap metallic phase can be described in two independent quasiparticles.
\end{abstract}

\section{INTRODUCTION}

Quantum spin systems with geometrical frustration have attracted considerable attention recently. There are a number of typical materials, such as the spingap compound $\mathrm{SrCu}_{2}\left(\mathrm{BO}_{3}\right)_{2} 1$ with the orthogonal-dimer structure 2 , 4 the pyrochlore compounds with the lattice of ions, 5.6. 0 , 9.10 .11 etc.

Besides frustrated spin systems studied intensively so far, metallic systems with geometrical frustration have attracted much interest. For instance, it has been claimed that frustration due to a tetrahedral network of $\mathrm{V}$ ions may be important to understand heavyfermion behavior of the metallic pyrochlore compound $\mathrm{LiV}_{2} \mathrm{O}_{4} .12$ Also, another prototypical pyrochlore compound $\mathrm{Y}(\mathrm{Sc}) \mathrm{Mn}_{2} 13$ shows spin-liquid behavior in a metallic phase.

Stimulated by these experimental findings, we study here the hole-doping effects on fully frustrated spin systems. To this end, we consider a simplified model in one dimension (1D), which still possesses the essence of strong frustration in the above-mentioned systems. To be precise, we employ the $t$ - $J$ ladder model with the orthogonal dimer structure, which also has linked-tetrahedra as a key structure. The advantage to employ the 1D model is that this simplification allow us to accurately calculate physical quantities with use of such a reliable numerical method as the density matrix renormalization group (DMRG), 14 and thus precisely study the effects of frustration on hole-doped systems.

This paper is organized as follows. In Sec. II, we introduce the frustrated $t$ - $J$ ladder model, and then in Sec. III study a metal-insulator transition upon hole doping. By computing spin correlation functions, we clarify the origin of the first-order phase transition found there. In Sec. IV, spin excitations in a metallic phase are discussed in detail. It is clarified to what extent a spin-gap metallic phase persists around the insulating phase. In particular, we show that a spin triplet excitation in the spin-gap phase can be described in terms of two independent quasiparticles. We obtain the phase diagram in Sec. $\mathrm{V}$, and summary and discussions are given in Sec. VI.

\section{FRUSTRATED LADDER MODEL}

The model we treat here is a frustrated electron ladder which is an extension of the spin-1/2 Heisenberg model with the orthogonal-dimer structure introduced by Gelfand.15 The model is illustrated schematically in Fig. 1. It has two competing antiferromagnetic interactions $J_{1}$ and $J_{2}$, giving rise to strong geometrical frustration. In a hole-doped case, we use the $t$ - $J$ model where electron hopping is assumed to have two distinct values, as shown in Fig. 11. The Hamiltonian is thus given by

$$
H=-\sum_{(i, j), \sigma} t_{\alpha} c_{i \sigma}^{\dagger} c_{j \sigma}+\sum_{(i, j)} J_{\alpha}\left(\mathbf{S}_{i} \cdot \mathbf{S}_{j}-\frac{1}{4} n_{i} n_{j}\right)
$$

where the summation is taken over nearest neighbor bonds $(i, j)$ indicated by the thick and thin lines in Fig. 1. Here, $c_{i \sigma}$ is the annihilation operator of an electron with spin $\sigma$ at the $i$-th site and $n_{i}\left(S_{i}\right)$ is the electron number (spin-1/2) operator. As mentioned above, hopping $t_{i}$ (exchange coupling $\left.J_{i}\right)$ takes either $t_{1}$ or $t_{2}\left(J_{1}\right.$ or $\left.J_{2}\right)$. Note that doubly occupied state at each site are implicitly forbidden in the $t$ - $J$ model. 


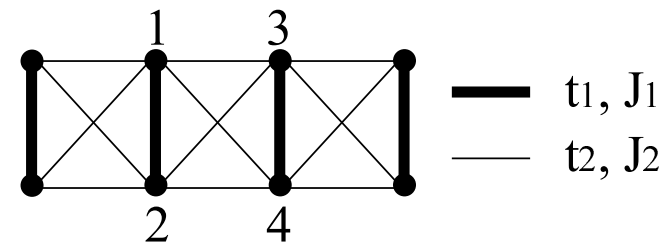

FIG. 1: Frustrated ladder system, which is often referred to as a linked-tetrahedra chain. The thick and thin lines correspond to the parameters $J_{1}\left(t_{1}\right)$ and $J_{2}\left(t_{2}\right)$, respectively.

We note here that the above model15. $16,17,18$ has several remarkable properties in addition to those for ordinary 1D frustrated spin systems: (i) it is a 1D analogue of the 2D orthogonal-dimer model (ShastrySutherland model23) relevant for $\mathrm{SrCu}_{2}\left(\mathrm{BO}_{3}\right)_{2}$, for which the ground state is exactly given by a direct product of decoupled dimers; (ii) it is also regarded as a $1 \mathrm{D}$ variant of the pyrochlore system (so-called linkedtetrahedra chain), which has frustrated tetrahedra as a key structure 15 A remarkable point related to (ii) is that, for noninteracting electrons on this lattice, the model has a dispersionless flat-band mode,

$$
\varepsilon(k)=\left\{\begin{array}{l}
t_{1} \\
-t_{1}-4 t_{2} \cos (k),
\end{array}\right.
$$

which reflects special geometry of the system, as is the case for the 3D pyrochlore lattice. Therefore, using this simplified model we can also explore the role of a flatband mode, which is important to understand properties of the pyrochlore system near half filling.

\section{METAL-INSULATOR TRANSITION}

Let us start our discussions with the ground-state properties of the $t$ - $J$ model in a metallic phase. We particularly focus on the nature of a metal-insulator transition induced by hole doping. Here, we use a variant of the infinite DMRG method, so-called-product-wavefunction renormalization group method 19 , to compute several quantities in a bulk limit.

In the undoped spin model, the system is either in the exact dimer-singlet phase $\left(J_{2} / J_{1} \in 0.71\right)$ or the plaquette-singlet phase $\left(J_{2} / J_{1}>0.71\right) .15$ We thus discuss the hole-doping effects on these phases separately. In the following analyses, we set $t_{2} / t_{1}=J_{2} / J_{1} \equiv \gamma$, and mainly show the results of $J_{1} / t_{1}=0.25\left(t_{1}=1.0\right)$ for simplicity.

\section{A. dimer phase}

We begin with the dimer phase, which is stabilized at half filling for the ratio of the exchange couplings, $\gamma<$
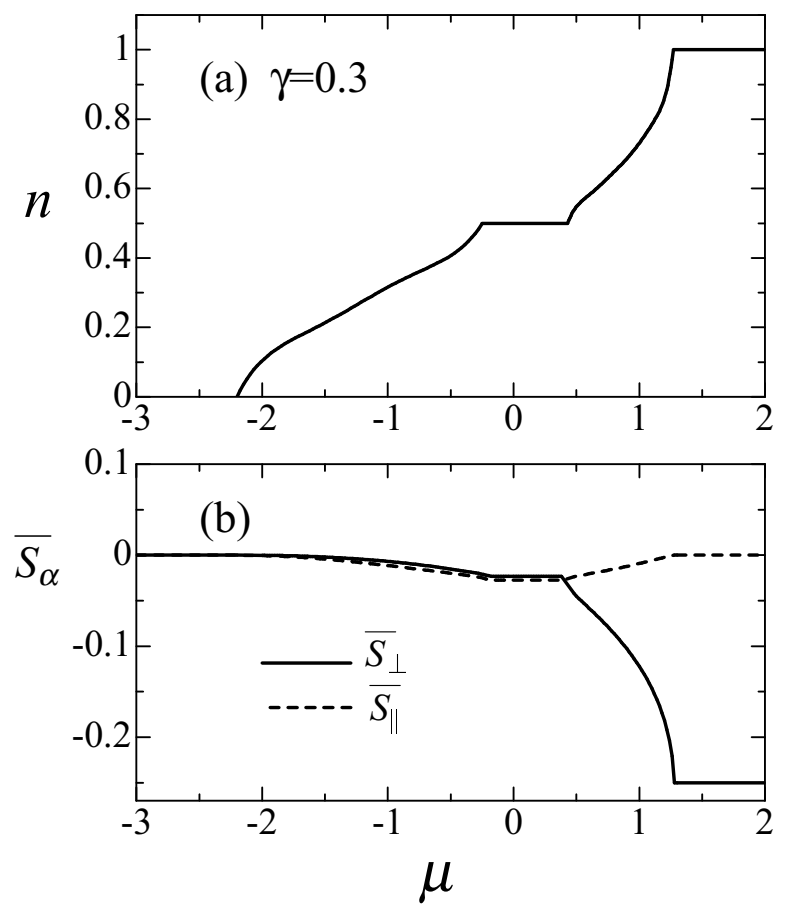

FIG. 2: (a) Electron density $n$ and (b) short-range spin correlations $\bar{S}_{\alpha}$ as a function of the chemical potential $\mu$ for $\gamma=0.3$ at $J_{1} / t_{1}=0.25$. The system is in a dimer-singlet insulating phase at half filling $n=1$.

0.71. The calculated electron density $n$ is shown in Fig. 2(a) as a function of the chemical potential $\mu$.

It is seen that hole-doping smoothly drives the system to a metallic phase. At quarter filling $(n=1 / 2)$, there appears a plateau in $n$, which is due to formation of a CDW insulating state. It is instructive to compare this smooth transition with first-order transitions found in the magnetization curve at half filling in the dimer phase:16 the magnetization exhibits jumps among the plateaus at zero, half, and full magnetization. The discontinuity in the magnetization reflect the fact that a triplet excited state in the dimer phase is completely localized due to the orthogonal-dimer structure. This is not the case for hole doping: doped holes are indeed heavy but still mobile, allowing a smooth transition to the metallic phase.

We next compute spin correlation functions, which are defined in the rung $(\perp)$ and chain $(\|)$ directions as,

$$
\begin{aligned}
\bar{S}_{\perp} & =\left\langle S_{1}^{z} S_{2}^{z}\right\rangle, \\
\bar{S}_{\|} & =\left\langle S_{1}^{z} S_{3}^{z}\right\rangle\left(=\left\langle S_{1}^{z} S_{4}^{z}\right\rangle\right) .
\end{aligned}
$$

Shown in Fig. 2(b) are the computed spin correlation functions. As the system approaches half filling from a metallic side, $\bar{S}_{\perp}$ along the rung rapidly decreases down to $-1 / 4$ whereas $\bar{S}_{\|}$along the chain becomes almost zero. Recall that the values of $\bar{S}_{\perp}=-1 / 4$ and $\bar{S}_{\|}=0$ are expected for a system with isolated dimers. Therefore, the above results of correlation functions imply that the sys- 

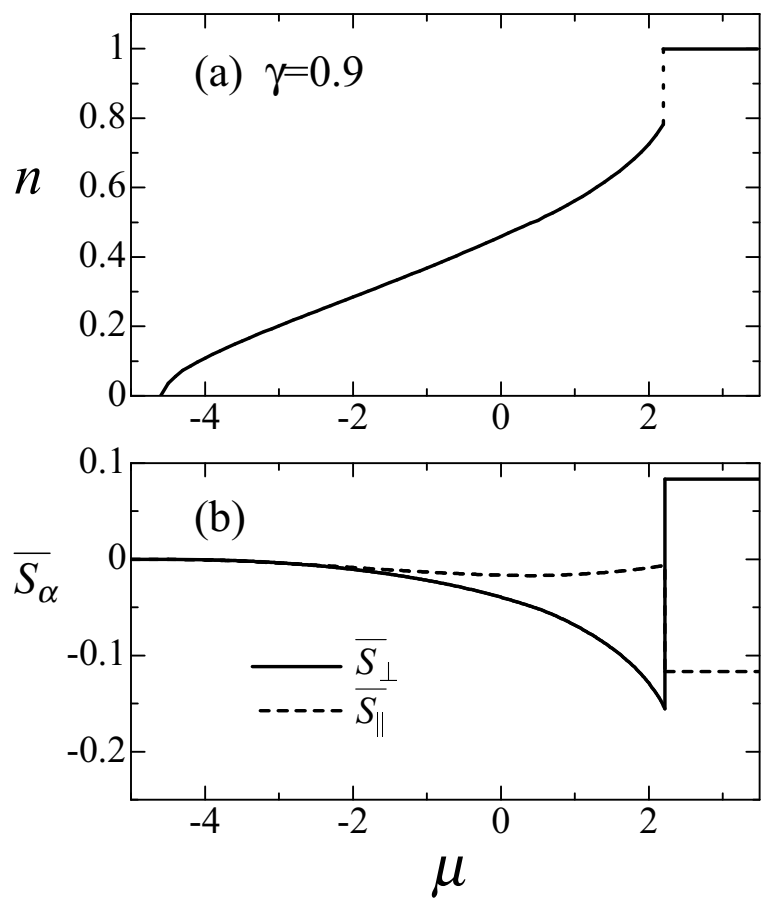

FIG. 3: (a) Electron density $n$ and (b) short-range spin correlations $\bar{S}_{\alpha}$ as a function of the chemical potential $\mu$ for $\gamma=0.9$ and $J_{1} / t_{1}=0.25$. This corresponds to hole doping in the plaquette phase. The dotted line in (a) indicates a jump associated with a first-order transition.

tem near half filling is regarded as a resonating metallic state of decoupled dimers.

\section{B. plaquette phase}

When holes are doped into the plaquette-singlet phase, the nature of a metal-insulator transition is completely changed. Shown in Fig. 3(a) is the electron density as a function of the chemical potential. It is remarkable that the system is driven to a metallic phase via a first-order phase transition, which is characterized by a jump in the electron density.

We can see the origin of the first-order metal-insulator transition by checking short-range spin correlation functions in the rung as well as leg directions, shown in Fig. 3(b). At half filling, $\bar{S}_{\perp}>0$ and $\bar{S}_{\|}<0$, being consistent with the plaquette-singlet ground state. However, once the system is driven to a metallic phase via the first-order transition, both of $\bar{S}_{\perp}$ and $\bar{S}_{\|}$become negative. Furthermore, when the system approaches halffilling from a metallic side, $\bar{S}_{\perp}$ decreases with negative values while $\bar{S}_{\|}$gets very small. Namely, in a metallic phase close to half filling dimer correlations along the rung are largely enhanced. Hence, in the vicinity of half filling, this dimer-dominant state strongly competes with the plaquette-singlet state energetically, giving rise to the first-order phase transition observed above.

We note here that such a first-order metal-insulator transition only occurs when holes are doped in the plaquette phase, but not in the dimer phase. It is also instructive to compare this with the results known for the magnetization curve at half filling, where first-order phase transitions occur only in the dimer phase $(\gamma<$ 0.71). 16 These apparently different aspects in phase transitions come from the fact that hole doping induces a competition between the dimer- and plaquette-singlet states, whereas a magnetic field induces a competition between the dimer- (or plaquette-) singlet state and the triplet state.

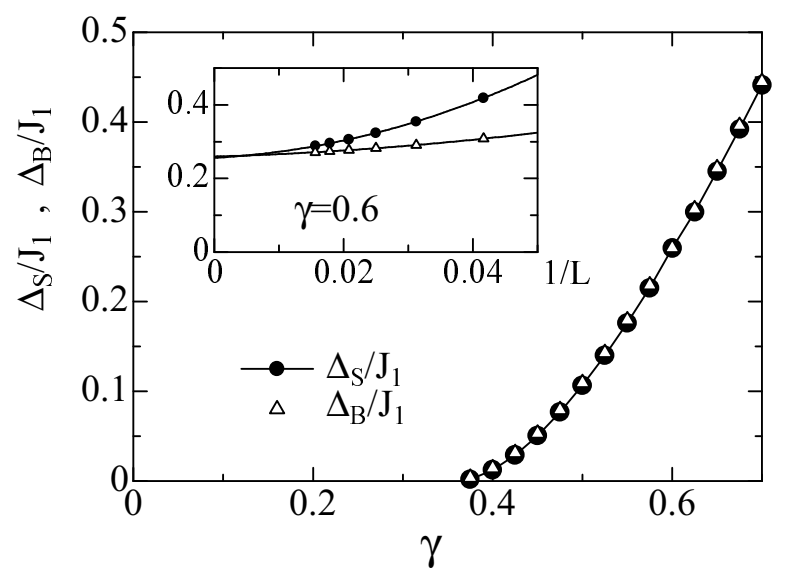

FIG. 4: Spin gap $\Delta_{S}$ and binding energy $\Delta_{B}$ of two holes $\left(N_{h}=2\right)$ for $J_{1} / t_{1}=0.25$. Inset shows a finite-size scaling analysis of $2 \times L$ systems for $\gamma=0.6$. 

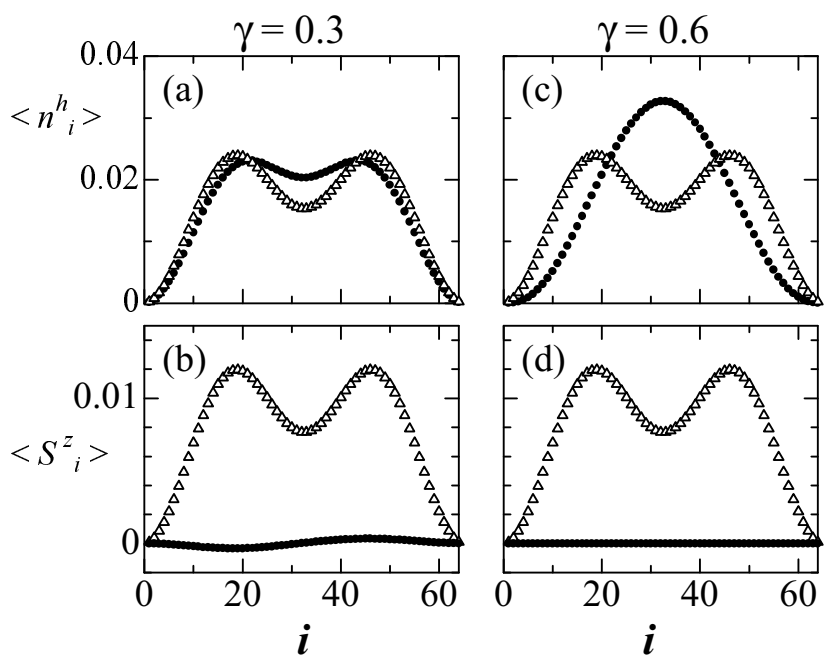

FIG. 5: Hole density $\left\langle n_{i}^{h}\right\rangle$ and spin density $\left\langle S_{i}^{z}\right\rangle$ for two holes on the $2 \times 64$ system: $\gamma=0.3$ for (a) and (b) while $\gamma=0.6$ for (c) and (d). Filled circles and open triangles correspond to the ground state and excited states, respectively.

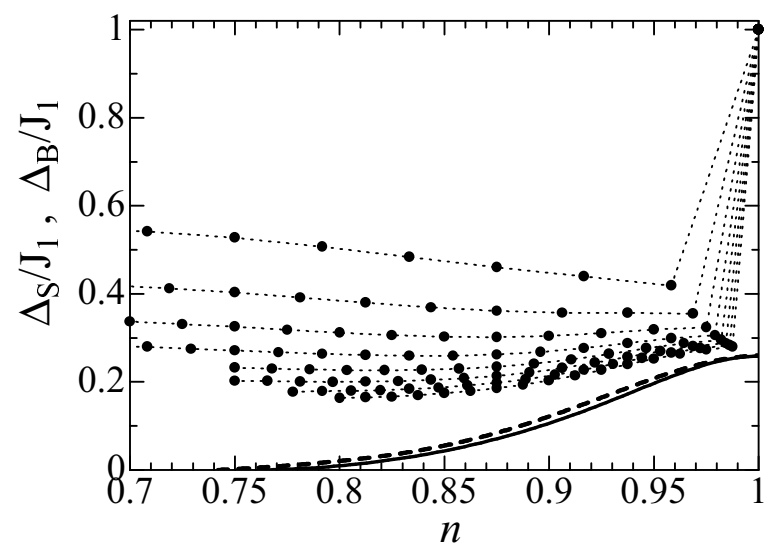

FIG. 6: Spin gap (solid line) and binding energy (broken line) as a function of $n\left(=1-N_{h} / L\right): \gamma=0.6$ and $J_{1} / t_{1}=0.25$. The dotted lines with filled circles are the results of $\Delta_{S}$ for $L=24,32,40,48,56,64,72,80$ from top to bottom.

\section{SPIN EXCITATIONS IN A METALLIC PHASE}

We now discuss spin excitations in a hole-doped system near half filling. We start with low-energy excitations in a system with two holes. As mentioned above, the system is driven to a metallic phase immediately upon hole doping for the dimer phase $(0<\gamma<0.71)$. On the other hand, for the plaquette phase $(\gamma>0.71)$, doped holes may be in a localized state, as a result of a first-order transition. Namely, holes are accommodated in a degenerate dispersionless level up to a critical hole density. In the following, we thus pay our attention to the former region, $0<\gamma<0.7$, which is more interesting as far as the system with a small number of holes is concerned.

In order to discuss excitations in a doped case, we consider two quantities, i.e. the spin gap $\Delta_{S}\left(N_{h}\right)$ and the binding energy $\Delta_{B}\left(N_{h}\right)$ respectively defined for $N_{h}$ (even number) holes on $2 \times L$ lattice sites as,

$$
\begin{aligned}
\Delta_{S}\left(N_{h}\right)= & E_{0}\left(N_{h}, S^{z}=1\right)-E_{0}\left(N_{h}, S^{z}=0\right) \\
\Delta_{B}\left(N_{h}\right)= & 2 E_{0}\left(N_{h}-1, S^{z}=1 / 2\right)-E_{0}\left(N_{h}, S^{z}=0\right) \\
& -E_{0}\left(N_{h}-2, S^{z}=0\right) .
\end{aligned}
$$

In Fig. 4, we show the spin gap $\Delta_{S}$ and the binding energy $\Delta_{B}$ computed for two doped holes $\left(N_{h}=2\right)$. The inset shows a finite-size scaling of $\Delta_{S}$ and $\Delta_{B}$ for $\gamma=0.6$. It should be noticed that the spin gap and the binding energy monotonically increase as $\gamma$ increases from $\gamma \simeq$ 0.35 . It is seen that the spin gap $\Delta_{S}$ just coincides with the binding energy $\Delta_{B}$, implying that a pair-breaking excitation in a metallic phase may be described by two independent quasiparticles. It is to be noted that the above spin gap in a doped system is totally different from the spin gap defined at half filling, $\Delta_{S}=J_{1}$, as we will see below.

Shown in Fig. 5 is a spatial distribution of the hole density $\left\langle n_{i}^{h}\right\rangle\left(n_{i}^{h} \equiv 1-n_{i}\right)$ and the spin density $\left\langle S_{i}^{z}\right\rangle$ for two holes on a $L=64$ system. Here filled circles and open triangles show the results for the singlet ground state and for a triplet excited state, respectively. For $\gamma=0.6$, as shown by filled circles in Fig. 5(c), a hole-pair exists in the ground state, implying the existence of an attractive interaction between two holes in the ground state. For a triplet excitation, the bound state is broken and two quasiparticles appear, as seen from open triangles in Fig. 5 (c) and (d). On the other hand, the situation is somewhat different for $\gamma=0.3$, where the repulsive interaction seems to exist even in the ground state, as shown by filled circles in Fig. 5(a) and (b). We have checked that a repulsive interaction in the latter case is mainly caused by finite-size effects. Therefore, it is expected that such a repulsive interaction becomes small for large $L$, and in the thermodynamic limit, doped holes may behave as two independent quasiparticles in the case of $\gamma=0.3$.

We now turn to the case with a finite density of holes. In Fig. 6, we show the spin gap as a function of the electron density $n$ for $\gamma=0.6$. The dotted lines with filled circles are the results of $\Delta_{S}$ for finite-size systems. It is seen that the value of the spin gap dramatically changes between half-filling and two-hole case, and then smoothly changes in the region $N_{h} \geq 2$. We have performed a finite-size scaling analysis of the spin gap from the data shown by dotted lines, obtaining the curve shown by the solid line. Note that the spin gap has a discontinuity at half filling, implying that the spin gap in a metallic phase has a different origin from the half-filled case. Also shown by the broken line is the binding energy $\Delta_{B}$ 
computed by a similar finite-size scaling analysis. Since $\Delta_{S}$ is nearly equal to $\Delta_{B}$, we can say that excited states may be described by two independent quasiparticles, as we have already seen in a system with two holes.

In order to see the nature of the attractive interaction between holes more clearly, we compute short-range holehole correlation functions $\bar{H}_{\alpha}$ in a bulk limit, which are defined in the rung $(\perp)$ and chain $(\|)$ directions,

$$
\begin{aligned}
\bar{H}_{\perp} & =\left\langle n_{1}^{h} n_{2}^{h}\right\rangle-\left\langle n^{h}\right\rangle^{2}, \\
\bar{H}_{\|} & =\left\langle n_{1}^{h} n_{3}^{h}\right\rangle-\left\langle n^{h}\right\rangle^{2} .
\end{aligned}
$$

In Fig. 78, we show $\bar{H}_{\alpha}$ as a function of $n$ for $\gamma=0.3$ and 0.6. For $\gamma=0.6$, there is the region with both of $\bar{H}_{\perp}$ and $\bar{H}_{\|}$being positive, where an effective interaction between holes is attractive in both directions. On the other hand, for $\gamma=0.3$, both of $\bar{H}_{\perp}$ and $\bar{H}_{\|}$are negative (i.e. repulsive interaction) in the whole range shown in the figure. By comparing these results with Fig. 1, we see that the region, where holes attract each other, roughly corresponds to the spin-gap metallic region. This means that the mechanism of spin-gap formation in a metallic phase, which is different from that in the insulating phase, is closely related to the attractive force between holes.

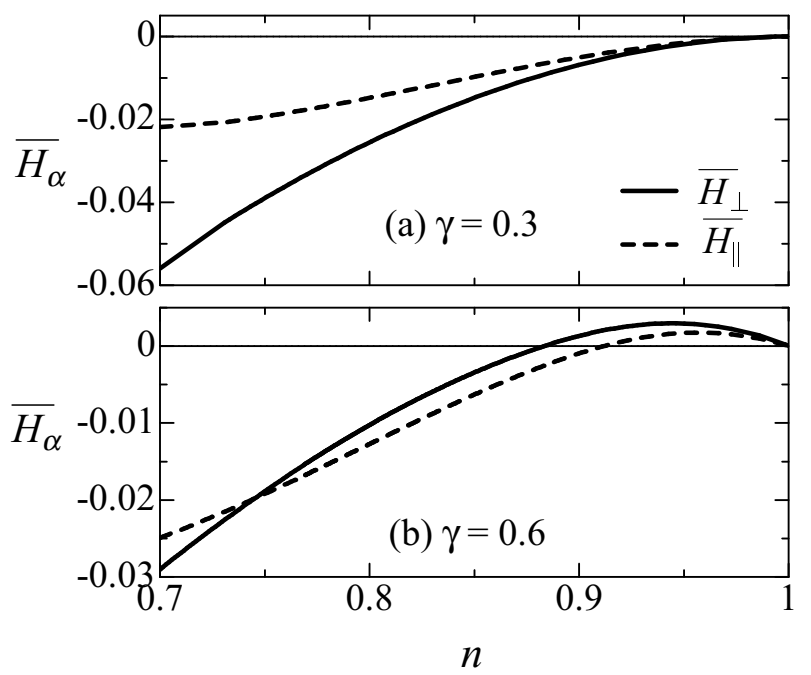

FIG. 7: Short-range hole-hole correlations $\bar{H}_{\alpha}$ for $\gamma=0.3,0.6$ and $J_{1} / t_{1}=0.25$.

\section{PHASE DIAGRAM}

Based on the above results, we now obtain the groundstate phase diagram, which is shown in Fig. 8. As found by Gelfand, 15 the ground state at half filling is either in the dimer-singlet (or plaquette-singlet) phase with spin gap at half filling for $\gamma<0.71(\gamma>0.71)$. These two phases are separated via a first-order phase transition when $\gamma$ is changed.
We have found that the transition from the insulating plaquette phase to a metallic phase is of first-order, being contrasted to the dimer phase showing a secondorder transition. Furthermore, a spin-gap metallic phase, which is indicated by the shaded region in Fig. 8, persists in the vicinity of half-filling, which is indeed caused by strong frustration. For the value $J_{1} / t_{1}=0.25$ shown in Fig. 8, we have found that a spin-gap metallic phase appears in the region of $\gamma>0.35\left(\equiv \gamma_{S}\right)$.

When the electron density is further decreased, we encounter a CDW insulating phase at quarter filling, characterized by the plateau in $n$ - $\mu$ curve. The region of a CDW phase becomes narrower as $\gamma$ increases, and finally vanishes beyond a certain critical value. As is seen from Fig. 2(b) and Fig. 3(b), a metallic phase continuously spreads in the whole region of $\gamma$, where short-range spin correlations along the rung, $\bar{S}_{\perp}$, always take negative values. This implies that dimer correlations are dominant for the ground state in a metallic phase, as is usually the case for a doped antiferromagnetic ladder. However, it should be noticed that in a metallic phase close to the plaquette-singlet insulator, a competition between the dimer- and plaquette- singlet states may give rise to dual properties in physical quantities.

We have thus far shown the calculated results by choosing a specific ratio of hopping and exchange. It is necessary to check what is changed when we take other choices of the parameters. For instance, we show the spin gap of a system with two holes in Fig. 9 when the ratio of $J_{1} / t_{1}$ is changed. As $J_{1} / t_{1}$ decreases, the critical point, $\gamma_{S}$, where the spin gap starts to develop, is shifted to a larger value. Note, however, that $\gamma_{S} \rightarrow 0.5$ in the limit of $J_{1} \ll t_{1}$. Namely, even if $J_{1} / t_{1}$ is small, a spin-gap metallic phase appears at least in the region of $0.5<\gamma<0.71$. This feature is different from the case of the $t$ - $J$ ladder without frustration, 20.21 22, 23.24.25 which has a spin gap only for relatively large $J_{1} / t_{1}$. 


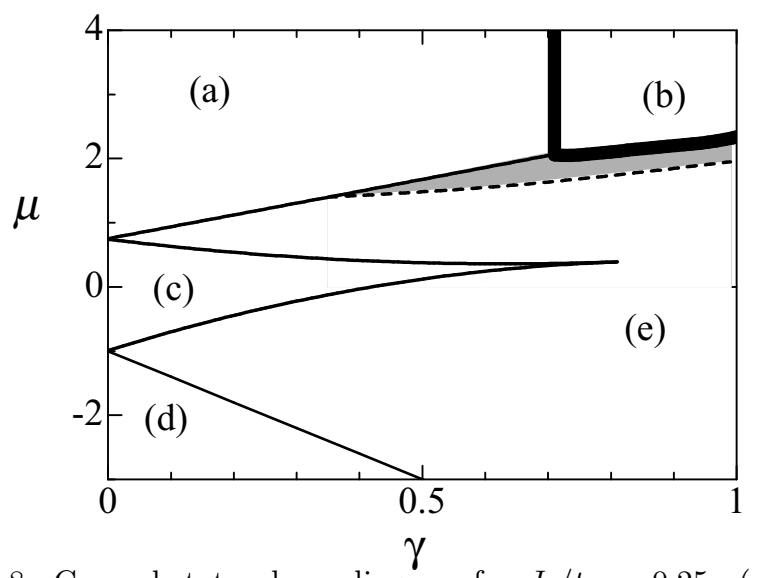

FIG. 8: Ground-state phase diagram for $J_{1} / t_{1}=0.25$ : (a) dimer-singlet phase $(n=1),(\mathrm{b})$ plaquette-singlet phase $(n=$ $1)$, (c) CDW phase $(n=1 / 2),(\mathrm{d})$ empty phase $(n=0),(\mathrm{e})$ metallic phase, and shaded area indicates a spin-gap metallic phase.

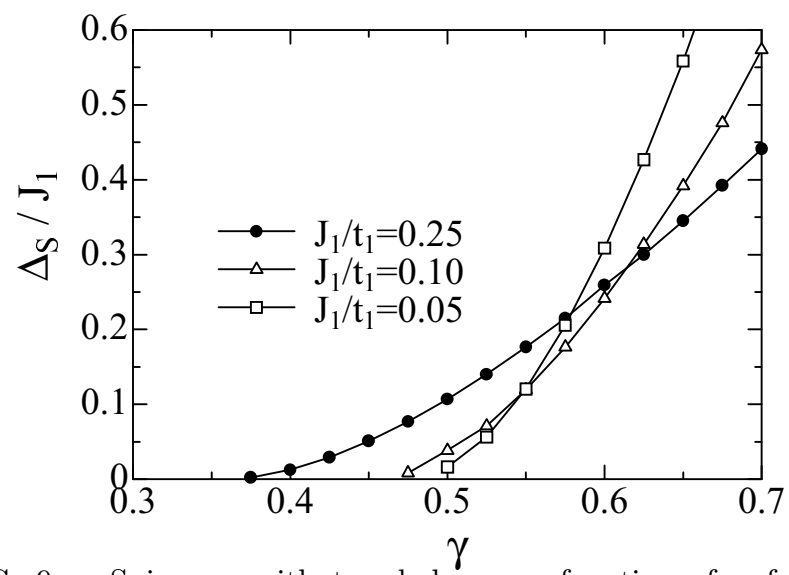

FIG. 9: Spin gap with two holes as a function of $\gamma$ for $J_{1} / t_{1}=0.25,0.10,0.05$. Other parameters are chosen as in Fig. 1 .

\section{SUMMARY AND DISCUSSIONS}

We have studied the hole-doping effects on a spin ladder system with the orthogonal-dimer structure, which also possesses linked-tetrahedra as a key structure. We have investigated how a metal-insulator transition is affected by geometrical frustration, by computing the electron density and several correlation functions by means of the DMRG method.

It has been found that when the system is in the dimer phase, hole-doping smoothly drives the system to a metallic phase. However, when the system is in the plaquette phase, a first-order transition occurs upon hole doping. The latter first-order transition occurs as a consequence of a strong competition between the dimerand plaquette-singlet states. This is different from ordinary metal-insulator transitions for a frustrated $t-J$ chain with next-nearest neighbor exchange couplings studied so far.26

The first-order phase transition found here is closely related to unique geometry of the linked-tetrahedra lattice, which causes a dispersionless (spatially isolated) mode, as mentioned in the beginning of the paper. It is to be noted that such a dispersionless mode caused by special geometry exists for the 3D pyrochlore lattice and also for the $2 \mathrm{D}$ orthogonal-dimer lattice. In this sense, the present results may capture some of characteristic properties common in this class of fully frustrated systems with doped holes. For instance, a pyrochlore antiferromagnetic system, indeed shows a competition between the dimer and plaquette phases, being similar to the present results. Therefore, we think that unusual properties may appear in a metallic phase of the doped pyrochlore system around the insulating phase. This is consistent with the results deduced by preservative analyses. 11 We also expect that such peculiar properties may emerge even in $2 \mathrm{D}$ orthogonal-dimer systems when holes are doped into an insulating phase around the boundary between the dimer and plaquette phases. 1 Detailed theoretical studies on hole-doped pyrochlore systems as well as 2D orthogonaldimer systems are left as an interesting open problem.

\section{Acknowledgements}

This work was partly supported by a Grant-in-Aid from the Ministry of Education, Science, Sports and Culture of Japan. A part of computations was done at the Supercomputer Center at the Institute for Solid State Physics, University of Tokyo and Yukawa Institute Computer Facility. A. Kawaguchi was supported by Japan Society for the Promotion of Science.
${ }^{1}$ H. Kageyama, K. Yoshimura, R. Stern, N. V. Mushnikov, K. Onizuka, M. Kato, K. Kosuge, C. P. Slichter, T. Goto and Y. Ueda, Phys. Rev. Lett. 82 (1999) 3168; J. Phys. 
Soc. Jpn. 68 (1999) 1821.

2 B. S. Shastry and B. Sutherland, Physica B 108 (1981) 11069.

3 S. Miyahara and K. Ueda, Phys. Rev. Lett. 82 (1999) 3701.

4 A. Koga and N. Kawakami, Phys. Rev. Lett. 84 (2000) 4461.

5 A. B. Harris, A. J. Berlinsky and C. Bruder, J. Appl. Phys. 69, 5200 (1991).

6 B. Canals and C. Lacroix, Phys. Rev. Lett. 80, 2933 (1998); Phys. Rev. B 61, 1149 (2000).

7 M. Isoda and S. Mori, J. Phys. Soc. Jpn. 67, 4022 (1998).

8 A. Koga and N. Kawakami, Phys. Rev. B63 (2001) 144432.

${ }^{9}$ H. Tsunetsugu, J. Phys. Soc. Jpn. 70 (2001) 640.

10 Y. Yamashita and K. Ueda, Phys. Rev. Lett. 85 (2000) 4960.

11 S. Fujimoto, Phys. Rev. B64 (2001) 085102.

12 S. Kondo, D. C. Johnston, C. A. Swenson, F. Borsa, A. V. Mahajan, L. L. Miller, T. Gu, A. I. Goldman, M. B. Maple, D. A. Gajewski, E. J. Freeman, N. R. Dilley, R. P. Dickey, J. Merrin, K. Kojima, G. M. Luke, Y. J. Uemura, O. Chmaissem and J. D. Jorgensen, Phys. Rev. Lett. 78, 3729 (1997).

13 R. Ballou, E. Leliévre-Berna and B. Fåk, Phys. Rev. Lett. 76, 2125 (1996).

14 S. R. White, Phys. Rev. Lett. 69, (1992) 2863; Phys. Rev. B. 48, (1993) 10345.
15 M. P. Gelfand, Phys. Rev. B. 43, (1991) 8644.

16 A. Honecker, F. Mila and M. Troyer, Eur. Phys. J. B. 15, (2000) 227.

17 W. Brenig and K. W. Becker, Phys. Rev. B. 64, (2001) 214413.

18 E. H. Kim, G. Fáth, J. Sólyom, and D. J. Scalapino, Phys. Rev. B. 62, (2000) 14965.

19 T. Nishino and K. Okunishi, J. Phys. Soc. Jpn. 64, (1995) 4084; Y. Hieida, K. Okunishi and Y. Akutsu, Phys. Lett. A. 233, (1997) 464.

${ }^{20}$ H. Tsunetsugu, M. Troyer and T. M. Rice, Phys. Rev. B 49 (1994) 16078.

21 E. Dagotto, J. Riera and D. Scalapino, Phys. Rev. B 45 (1992) 5744.

22 S. R. White and D. J. Scalapino, Phys. Rev. B 55 (1997) 6504 .

23 T. Siller, M. Troyer, T. M. Rice and S. R. White, Phys. Rev. B 63 (2001) 195106.

24 D. Poilblanc, O. Chiappa, J. Riera, S. R. White and D. J. Scalapino, Phys. Rev. B 62 (2000) R14633.

25 C. A. Hayward, D. Poilblanc, R. M. Noack, D. J. Scalapino and W. Hanke, Phys. Rev. Lett. 75 (1995) 926.

26 M. Ogata, M. U. Luchini, T. M. Rice, Phys. Rev. B 44 (1991) 12083. 